\title{
MUTATION LOAD AND THE SURVIVAL OF SMALL POPULATIONS
}

\author{
MiCHAEL LYNCH \\ Department of Biology, University of Oregon, Eugene, OR 97403 USA \\ AND \\ WILFRIED GABRIEL \\ Department of Physiological Ecology, Max Planck Institute for Limnology, \\ Postfach 165, D-2320 Plön, FEDERAL REPUBLIC OF GERMANY
}

\begin{abstract}
Previous attempts to model the joint action of selection and mutation in finite populations have treated population size as being independent of the mutation load. However, the accumulation of deleterious mutations is expected to cause a gradual reduction in population size. Consequently, in small populations random genetic drift will progressively overpower selection making it easier to fix future mutations. This synergistic interaction, which we refer to as a mutational melt-down, ultimately leads to population extinction. For many conditions, the coefficient of variation of extinction time is less than 0.1 , and for species that reproduce by binary fission, the expected extinction time is quite insensitive to population carrying capacity. These results are consistent with observations that many cultures of ciliated protozoans and vertebrate fibroblasts have characteristic extinction times. The model also predicts that clonal lineages are unlikely to survive more than $10^{4}$ to $10^{5}$ generations, which is consistent with existing data on parthenogenetic animals. Contrary to the usual view that Muller's ratchet does more damage when selection is weak, we show that the mean extinction time declines as mutations become more deleterious. Although very small sexual populations, such as self-fertilized lines, are subject to mutational meltdowns, recombination effectively eliminates the process when the effective population size exceeds a dozen or so. The concept of the effective mutation load is developed, and several procedures for estimating it are described. It is shown that this load can be reduced substantially when mutational effects are highly variable.
\end{abstract}

Received June 27, 1989. Accepted January 17, 1990.

All populations are doomed to eventual extinction. The reasons include temporal variation in the environment, demographic stochasticity (random variation in survivorship, birth rate, and sex ratio), and genetic problems such as inbreeding depression, the loss of adaptive variation by random drift, and recurrent deleterious mutations (Soulé, 1987; Lande, 1988). This paper is concerned exclusively with the latter problem. Deleterious mutations impose a load on populations through the reduction in the mean survivorship and/or reproductive rates of individuals (Haldane, 1937; Muller, 1950; Wallace, 1987). Ultimately, this load should be manifested in a higher likelihood of population extinction.

It has long been known that in effectively infinite populations an equilibrium mutation load exists that is independent of the mutational effect (Haldane, 1937; Muller, 1950; Hopf et al., 1988). Depending on the degree of dominance, the reduction in mean fitness per locus is between once and twice the gametic mutation rate. Nei (1968) obtained the same result for recessive lethals in small populations. On the other hand, Kimura et al. (1963) argued that the load due to mildly deleterious mutations is inversely proportional to population size, approaching the selection coefficient in the absence of back-mutation.

These results for sexual populations in drift-mutation-selection balance may be contrasted with Muller's (1964) idea that a mutational ratchet operates in asexual populations, continually driving them to lower and lower fitness. The idea here is that, in the absence of sex, no genotype can ever produce an offspring with fewer mutations than its own load. In a finite population, there is always a possibility that the class of individuals with the lowest mutational load will not leave any offspring. Each time this happens, the ratchet clicks. Random fixation of deleterious alleles also occurs in sexual populations, but the ratchet can be slowed considerably since parents can produce offspring with reduced mutation loads (Felsenstein, 1974; Maynard Smith, 1978; Heller and Maynard Smith, 1979; Pamilo et al., 1987; Bell, 1988; Kondrashov, 1988). 
Little formal work has been done in this area, and verbal arguments are frequently made that recombination essentially silences the ratchet (e.g., the majority of papers in Michod and Levin, 1988).

A peculiar feature of all of the existing models of the mutation load is that, despite their deleterious nature, the mutations have no influence on the demographic features of the populations that harbor them. Selection acts on the relative contributions of individuals to the next generation, but population-level fertility is assumed to be effectively infinite. The number of reproducing adults is fixed, and the population is restored to its carrying capacity each generation. There is no population extinction.

Our purpose is to evaluate the degree to which deleterious mutations influence the persistence times of small populations. Assuming that the mutation load influences viability prior to reproduction, and employing empirical estimates of the rates and effects of such mutations, we show that only extremely large asexual lineages are likely to survive for more than $10^{4}-10^{5}$ generations, unless back-mutations are much more common than currently believed. For sexual organisms, the viability load is a significant source of extinction only in very small populations.

Muller's ratchet causes a sort of mutational melt-down. As the mutation load builds up, there is a gradual reduction in population size. This increases the relative importance of random drift as an evolutionary force, which enhances the chances of fixation of future deleterious mutations, ultimately leading to extinction. There can be no equilibrium mutation load or population size unless the time to extinction is great enough to allow a substantial likelihood of back-mutation.

\section{The Model}

The extinction process that we are concerned with is not easily solved analytically since it involves a double-diffusion process for individual fitness and population size. Therefore, we have relied heavily on computer simulation. The populations under consideration are assumed to be diploid and either asexual (ameiotic) or monoecious with random mating and free recombination. The schedule of events in the life cycle is mutation $\rightarrow$ differential survival $\rightarrow$ reproduction. In all simulations, the initial population was assumed to be completely free of deleterious genes. Survivorship to maturity was assumed to be perfect for mutation-free genotypes, i.e., there are no environmental sources of mortality. Since neither condition is expected to be met in nature, our estimates of population longevity can be viewed as upper limits.

The mutation rate per individual $(\mu)$ is assumed to be Poisson distributed, and all new mutations are assumed to arise at unique loci. Initially, we assume that backmutations are of negligible importance, but we will relax this restriction later in the paper. The mutations act additively within loci, but the locus-specific fitnesses act independently. An individual's probability of survival is therefore $W=\prod_{i=1}^{n}\left(1-s_{i}\right)$ where the $s_{i}$ are the selection coefficients at the $n$ loci. Our treatment ignores the class of lethal recessive mutations, again causing our estimates of population longevity to be on the liberal side. However, the influence of recessive lethals on the process we are studying is expected to be small. At least in the case of Drosophila, the mutation rate to recessive lethals is approximately 30 times less than that to mildly deleterious alleles (Crow and Simmons, 1983), and the likelihood of fixation of a recessive lethal is very small even in tiny populations.

A simple form of population regulation is assumed throughout. Each surviving individual has the capacity to be a mother to an average of $R$ offspring. However, the environment can support the production of only $K$ zygotes. Thus the population size in the next generation is $N^{\prime}=R N$ if $R N<K$, otherwise $N^{\prime}=K$, where $N$ is the number of survivors in the preceding generation. The survivorship of each individual is evaluated by drawing a uniformly distributed random variable in the range of 0 to +1 and comparing it to the individual's probability of survival. Zygotes (asexuality) or gametes (sexuality) are randomly drawn from the pool of survivors. Population extinction requires mortality of all $N^{\prime}$ individuals. By iterating the above process over many rep- 
licate populations with fixed $\mu, s, R$, and $K$, information is obtained on the theoretical distributions of extinction times.

This idealized model can also be used to estimate the mutation load in experimental populations. Suppose a set of replicates with identical $R$ and $K$ is followed until extinction. One can then inquire as to the combination of $\mu$ and $s$ that best explains the observed distribution of extinction times. To accommodate nongenetic sources of mortality, the expression for individual survivorship can be multiplied by a constant $W_{0}<1$, which can also be estimated from the retrospective analysis.

It seems likely that aspects of empirical populations will deviate from the assumptions of the model. The mutational effect is likely to be variable rather than constant, locus-specific fitnesses may not interact multiplicatively, mutations may vary in their degree of dominance, and some mutations may be beneficial. However, there are currently no empirical methods to evaluate the magnitude of these problems, and for mutations with small effects, it is unlikely that this will change in the near future. We therefore refer to the product $\mu s$ estimated by this sort of analysis as the effective mutation load. In other words, the effective mutation load is the $\mu s$ of an ideal set of populations that yields extinction properties most compatible with the observed (possibly nonideal) set. In spirit, this approach is similar to the use of measures of effective population size and effective numbers of alleles and loci in population genetics (Crow and Kimura, 1970). The quantity $\mu s$ can be thought of as the proportional reduction in fitness due to deleterious mutations appearing in the preceding generation. We will show below that for the small $K(\leq 5)$ that are ideal for estimating the mutation load in laboratory experiments, the time to extinction depends only on the product $\mu s$.

\section{EMPIRICAL ESTIMATES OF THE MUTATION LOAD}

Before embarking on a fuller analysis of the mutational melt-down, we first derive some estimates of the effective mutation load in order to put some bounds on the values of $\mu$ and $s$ that ought to be explored. At the onset, we note that the only sexual species for which data are available is Drosophila melanogaster. Mutation accumulation experiments with this species have been numerous and often quite large. The data, which have already been reviewed extensively by Crow and Simmons (1983), indicate that $\mu \simeq 0.6$ and $s \simeq 0.025$, ignoring lethals. In other words, an average gamete incurs approximately 0.3 new mutations/ generation, and each of these causes an approximately $2.5 \%$ reduction in viability $(5 \%$ in the homozygous state).

We now review several studies that were not designed explicitly for the estimation of the mutation load but nevertheless provide data that allow such an analysis.

\section{Ciliates}

The ciliated protozoa are well-known for the finite life-span of clones maintained in culture without recombination (reviews in Sonneborn, 1954, 1957; Nanney, 1974; Smith-Sonneborn, 1981). The literature is full of references associating particular species (strains) with characteristic numbers of cell fissions/clonal life span, usually in the range of 150-1,500 and with low intrastrain variance. Although a few clonal lineages have been described as immortal, this seems to be an exaggeration. Nuclear transplantation experiments indicate that clonal deterioration is caused by nuclear rather than cytoplasmic factors (Aufderheide, 1984, 1987).

Ciliates have a unique form of genetic organization. Most species contain a macronucleus and a micronucleus. The micronucleus replicates by mitosis during clonal propagation, but otherwise remains inactive. It undergoes meiosis during conjugation (out-crossing) and autogamy (self-fertilization), and following daughter-cell formation divides by mitosis, one product remaining as a micronucleus and another becoming the macronucleus. The macronucleus then undergoes rounds of replication until its DNA content is hundreds of times that of the micronucleus. It remains transcriptionally active throughout the period of clonal propagation. The mechanism of macronuclear division is poorly under- 


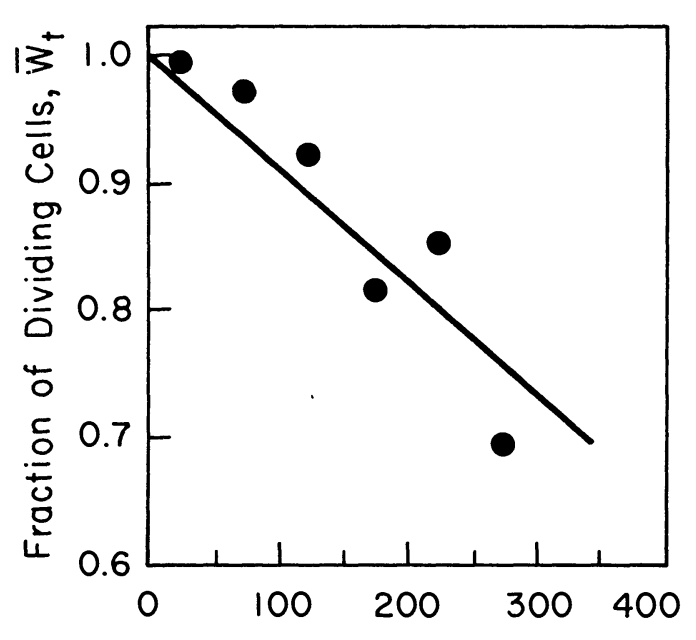

Cell Divisions, $\dagger$

Fig. 1. The fraction of reproductive cells in subclones $(K=3)$ of Paramecium caudatum, extracted from a stock culture. The line is the least-squares regression constrained to pass through $(0,1)$. The data are from Takagi and Yoshida (1980).

stood but is more along the lines of fragmentation than mitosis.

There are two ways to look at mutation accumulation in ciliates. First, from the standpoint of expressed fitness, the only relevant genetic deterioration during clonal propagation is that in the macronucleus. Such deterioration may include ordinary errors of replication, but due to the amitotic nature of macronuclear propagation, it can also include such extreme events as chromosome loss. Indeed, Kimura (1957) produced a stochastic model of chromosome loss that yields extinction dynamics that are reasonably consistent with the existing data.

Second, the consequences of micronuclear mutations become apparent only after a phase of sex. Thus the ciliates provide an ideal system for studying the mutational input of genetic load. Potentially deleterious mutations accumulate in the micronucleus in a neutral fashion between bouts of sexual reproduction.

Data from Takagi and Yoshida (1980) allow two estimates of the macronuclear mutation load for a clone (M18a) of Paramecium caudatum. Prior to analysis, the animals were maintained in a stock culture, and the data bear out the assumption that the population size was adequate for selec- tion to keep the mutation load at a low level. Starting at fission number 240,10 subclones were isolated from the mass culture, and each of these was subsequently maintained as three sublines with single-cell descent and with nondividing sublines being replaced by members of sister sublines whenever possible. With such small line sizes, selection will be ineffective against all but very deleterious mutations. Thus mean fitness (celldivision potential) at division $t$ may be approximated by $\bar{W}_{t}=(1-s)^{\mu t}$, which reduces to $\overline{W_{t}} \simeq 1-\mu s t$ for small $\mu$ st. The mutation load $(\mu s)$ can then be estimated by regression of the fraction of dividing cells on the number of cell divisions, under the constraint that the $y$-intercept is equal to 1 . The least-squares estimator of the slope of such a regression is $-\overline{\mu S}=\sum t\left[\overline{W_{t}}-1\right] / \sum t^{2}$. Using the data in the authors' Figure 4, we obtain $\widehat{\mu S}=0.00090$ (Fig. 1). Since there was some opportunity for selection to act among sublines (within subclones), this estimate of the mutation load is downwardly biased. The linear reduction of cell-division potential with time does not appear to be consistent with the notion among some authors that Paramecia have programmed cell death.

For this study, there is an alternative, less biased way to estimate the mutation load. For subclones maintained as $K=1,3,6$, and 15 sublines, Takagi and Yoshida (1980) recorded average life-spans of 142, 238, 303, and 386 cell divisions. It will be shown below that for such small $K$, mean extinction time depends primarily on the product $\mu s$. Employing the model described above, the four observed extinction times are found to be consistent with $\mu s=0.0017,0.00096$, 0.0016 , and 0.0017 , the average of which is 0.0014 .

Smith-Sonneborn (1979) maintained replicate lines of single cells of Paramecium tetraurelia, passing single offspring on at each fission and recording the time to extinction. For lineages that had already survived 40 , 80 , and 140 initial fissions, the total mean life-span was 114,143 , and 153 cell divisions. Taking this into account, and using our model with $K=R=1$, these extinction times are consistent with $\mu s=0.00013$, 0.00012 , and 0.00026 , which yields an average of 0.00017 . From survivorship data 
given in Smith-Sonneborn (1979, Figs. 1, 2, and 4), it is also possible to compute the mean probability of survival through each fission. Regression of these estimates on the number of past cell divisions yields a slope of -0.00031 . Averaging these two estimates of the mutation load, we obtain $\mu s \simeq$ 0.00024 .

Simon and Nanney (1979) provide data that allow estimation of the mutation load occurring in the micronucleus. They started with a series of inbred isolates of Tetrahymena thermophila and maintained them asexually in culture for over 10 years, while periodically subjecting them to a mating test. There was a substantial temporal decline in the viability of sexually produced progeny. The least-squares regression of this measure of fitness on years of clonal propagation, $\sum t \ln \left(W_{t}\right) / \sum t^{2}$ under the constraint that $W_{0}=1$, yields an estimate of $\ln \left[(1-s)^{\mu}\right]$ $\simeq-0.056$, which implies an annual $\mu \mathrm{s} \simeq$ 0.056 (data for 9 clones in the authors' Table $\mathrm{V}$, ignoring one outlier). To put this estimate of the annual mutation load for the micronucleus on a generational basis, we need to know the mean fission rate. This information is not available, but Nanney (pers. comm.) reasons that it was on the order of $50 /$ month. Taking this into consideration, an estimate of $\mu s \simeq 0.000093$ is obtained.

\section{Fibroblast Cultures}

As in the case of ciliates, laboratory cultures of fibroblasts are characterized by predictable life-spans. Many cell biologists regard the eventual loss of cell-division potential to be an adaptive, terminal phase of the developmental process, and there is indeed a correlation between in vitro fibroblast longevity and average life-span of the donor species (Hayflick, 1984). However, it is an open question as to whether developmental regulation in vitro is at all similar to that in vivo. We show below that as with ciliates, the loss of cell-division potential in culture is not synchronous, which seems contrary to expectations under tight developmental control. There is, however, a gradual accumulation of chromosomal abnormality and loss of DNA repair capacity and fidelity with culture age (Stanulis-Prae- ger, 1987). We now consider the magnitude of the mutation load required to account for the observed properties of cultured fibroblasts, without prejudice as to their adaptive and/or developmental significance.

We first consider a study performed by Smith and Pereira-Smith (1977) on human fibroblast cultures. Periodically, they extracted single cells from mass cultures, allowed them to grow for a short period of time, and recorded the number of cell divisions. Letting $W_{t}$ be the expected probability of division for a cell extracted from the mass culture at population doubling $t$ (the usual time reference in cell-culture studies), the probability of at least $x$ divisions $P_{x}(t)$ will be proportional to $W_{t}^{x}$. (This ignores the reduction of cell-division potential that is expected to occur throughout an assay, but the bias involved is not serious so long as the assay duration is short relative to the age of the mass culture, which is the case in the following analyses.) The cumulative probability distribution of cell divisions at time $t$ allows an estimation of $\bar{W}_{t}$. Fixing the expected probability of at least zero cell divisions at one, the least-squares estimate of $\ln \left(\overline{W_{t}}\right)$ is approximated by $\sum x$ $\ln \left[P_{x}(t)\right] / \sum x^{2}$. This approach yields good fits to the data obtained at four successive samples of the mass culture (Fig. 2). Clearly, there is a progressive decline in cell-division potential. Moreover, regression of $\bar{W}_{t}$ on $t$ reveals an essentially linear decline in celldivision potential throughout the period of study (Fig. 3), consistent with expectations for small $\mu s t$. The minimum mutation load, $\mu s$ estimated by the slope of this regression, is $0.0041\left(r^{2}=0.59\right)$.

Application of the above procedure to data on hamster (Raes and Remacle, 1983) and chicken (Angello and Prothero, 1985) fibroblasts yields qualitatively similar results, but somewhat higher mutation loads, $0.0055\left(r^{2}\right.$ $=0.78)$ and $0.012\left(r^{2}=0.47\right)$, respectively (Fig. 3). The intercept is significantly below $\overline{W_{0}}=1$ in the case of the hamster, suggesting a problem with culture conditions independent of culture age.

The data on the mouse (Karatza and Shall, 1984) in Figure 3 are derived from the same type of experiment discussed above. In this case, however, the fraction of nondividing cells was recorded as a function of popu- 


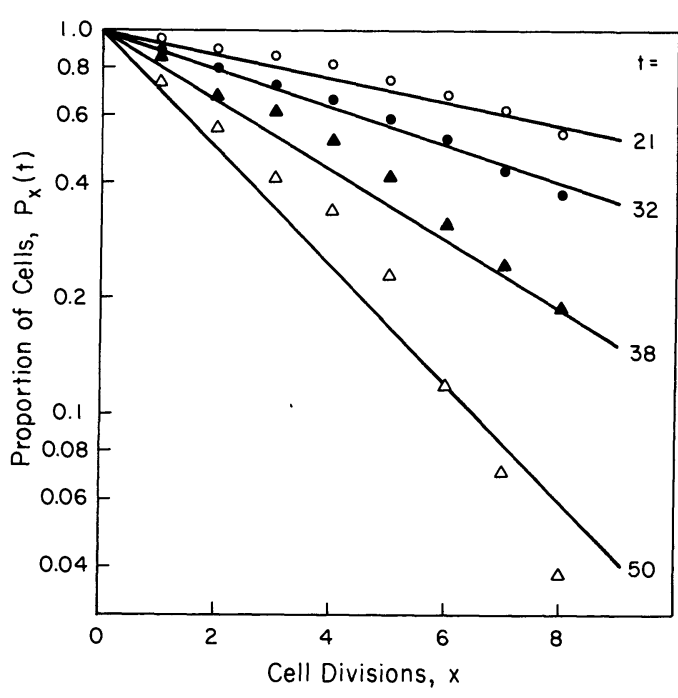

FIG. 2. The proportion of single-cell isolates dividing at least $x$ times at different levels of population doublings $(t)$. The data are for human fibroblasts in culture (Smith and Pereira-Smith, 1977). The straight lines are least-squares regressions.

lation doublings. One minus this quantity was taken to be an estimate of $\bar{W}_{t}$. Again, it can be seen that cell-division potential declines linearly with the number of cell divisions, the mutational load estimate being $0.020\left(r^{2}=0.70\right)$.

In summary, we find that the minimum mutation load for fibroblasts reproducing by mitosis ranges from 0.004 to 0.02 per cell division. The mean, 0.01 , is substantially higher than the mean for protozoans reproducing by mitosis $(0.005)$, but somewhat less than that for the Drosophila germ line $(0.015 /$ generation $)$. It is likely that the dominant mutational mechanisms vary substantially between these three groups.

Care should be taken in extrapolating the fibroblast data to the situation in vivo. On the one hand, culture conditions may be highly mutagenic relative to the natural experience of the cells. But on the other hand, many loci with essential functions in natural tissues may be dispensible in in vitro cultures.

\section{RNA Virus}

It has long been known that viral populations accumulate mutations rapidly when cultured by undiluted passage but maintain a high level of infectiousness when cultured

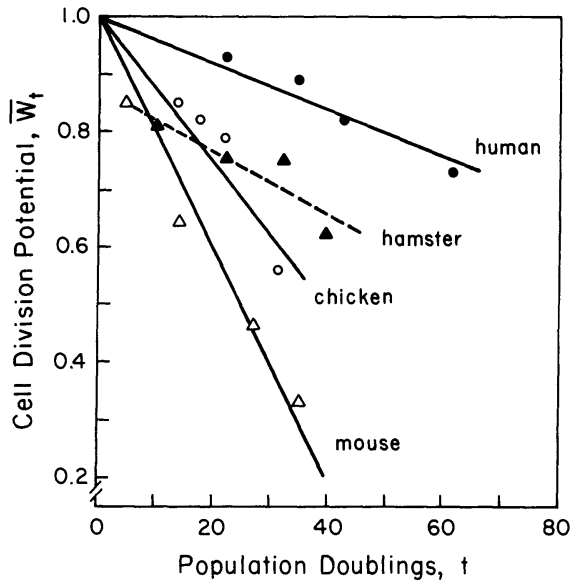

FIG. 3. Least-squares regressions of cell-division potential on population doubling for fibroblast cultures, $\bar{W}_{t}=1-\mu s t$. The $y$-intercepts were constrained to pass through $(1,0)$, except in the case of the hamster. Sources of data: human (Smith and Pereira-Smith, 1977), hamster (Raes and Remacle, 1973), chicken (Angelo and Prothero, 1985), and mouse (Karatza and Shall, 1984).

by dilute passage. Serial transfer of the viral population of a dilute level results in a small number of viral particles infecting each host cell. Undiluted passage results in a high multiplicity of infection. In the early stages of undiluted passage, mutations that reduce the infectivity of individual viruses tend to be nearly neutral-cell invasion is not impeded so long as another member of the coinfecting group is infective. Moreover, when the size of coinfecting groups is large, the variance in fitness among groups will be low, and this further reduces the ability of selection to eliminate deleterious mutations. Selection is minimal within groups since all inhabitants of a host cell share the same gene products for replication. Thus, the temporal reduction in individual infectivity under undiluted passage provides an estimate of the mutational load since the inability to infect can be equated to mortality under the conditions of dilute passage.

Data on influenza virus suggest an extraordinarily high mutation load in this organism. Von Magnus (1954, Fig. 1) presents data on the concentration of infectious particles at the beginning of four undiluted passages of PR8 virus in allantoic fluids of the chick embryo. Hemagglutinin assays performed at the same time provide estimates 
of total viral concentration (hemagglutinating units $\times 10^{6}$ ). The ratio of these two measures yields an estimate of the proportion of viruses capable of independent infection. Figure 4 shows that there is an approximately exponential decline in mean fitness with the number of population doublings (generation number). [Chao (1988) suggested that the reduction in infectivity accelerates with $t$ and that this indicates a synergistic interaction between successive mutations. However, with only one degree of freedom, the curvilinear regression cannot be regarded as significant.] The slope of the least-squares linear regression, -0.59 , provides an estimate of $\ln \left[(1-s)^{\mu}\right]$. This implies that $\mu s$ is on the order of 0.5 . Such a high rate is not totally unexpected since RNA viruses such as influenza are known to have an extremely high mutation rate due to the absence of repair mechanisms (see Nei, 1983 for review) and possibly to the properties of RNA polymerase.

\section{TIME TO EXTINCTION UNDER}

\section{RECURRENT DELETERIOUS MUTATION}

Taking the preceding empirical estimates of the mutation load as a guide to the possible parameter space of $\mu$ and $s$, we now consider in some detail the consequences of unconditionally deleterious mutations for population longevity. Acknowledging that $\mu s$ may be as high as 0.5 in some RNA viruses, we will limit our attention to the extremes of $\mu s=0.02$ to 0.0002 , which seems to be the approximate situation for eucaryotes. Since there are situations where the dynamics of extinction depend not just on the product $\mu s$, we will assume that the approximate range of $\mu$ is 0.3 to 1.2 new mutations per zygote, which is half to twice the estimate for Drosophila. We again emphasize that because of our assumption of zero pre-reproductive mortality in the absence of new mutations, our projected mean times to extinction $\left(\bar{t}_{E}\right)$ are upper limits. Initially, we will confine our attention to asexual populations.

The process of mutational melt-down is illustrated for a set of small $(K=32)$, high fecundity $(R \geq 32)$ asexual populations in Figure 5. With the Drosophila parameters $\mu$ $=0.6$ and $s=0.025$, there is an essentially linear increase in the mean number of mu-

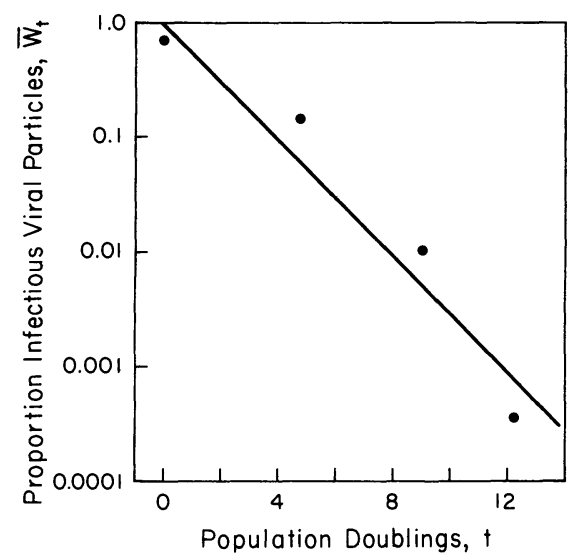

FIG. 4. Least-squares regression of infectivity on population doublings for influenza virus maintained by undiluted passage. The data are from von Magnus (1954).

tations/individual through time $(0.45 \mathrm{mu}$ tations/generation). Due to the high mutation pressure and the small population size, selection impedes the mutation accumulation process only by about $25 \%$, and the variance of the mutational load among replicate populations is quite low. This causes an exponential decline in the average survivorship of individuals. As mean individual survival drops below 0.25 , extinctions begin to occur, with virtually all populations being extinguished in the following 150 generations. The coefficient of variation of the times to extinction in this simulation is 0.13 , which is surprisingly small for an inherently stochastic process.

Figure 6 outlines the relationship of expected extinction time of $\mu, s$, and $K$ for the extreme situations of high $(R=K)$ and low $(R=2)$ fecundity. In the former case, a single surviving individual is sufficient to bring the population back up to the carrying capacity in the following generation. Several conclusions can be drawn from the simulation results. First, it appears rather unlikely that clonal lineages with $K<10^{4}$ or so will survive much more than $10^{4}$ generations. This result is qualitatively consistent with a large body of molecular data that suggests that most obligately parthenogenetic animals are phylogenetically young (Bell, 1982; Lynch, 1984).

Second, for fixed $\mu s$, the time to extinction decreases as the mutation rate increases. However, the effect is not very large pro- 


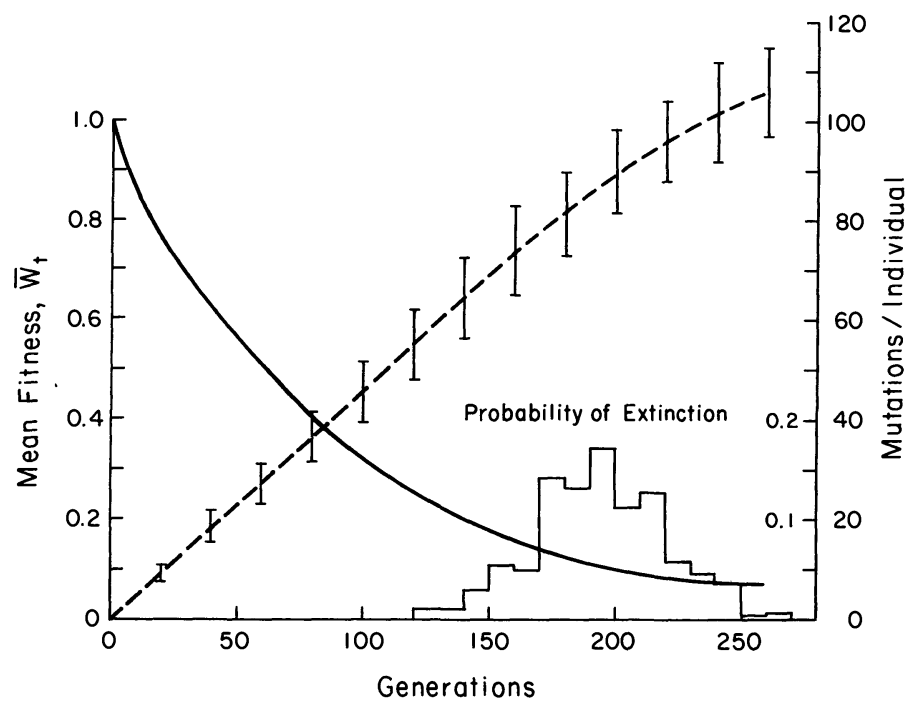

FIG. 5. The accumulation of mutations (dashed line) and reduction in individual survivorship (solid line) in asexual populations with $K=32, R \geq 32, \mu=0.6$, and $s=0.025$. The vertical lines denote \pm 1 SD. The histogram is the frequency distribution of extinction times for replicate populations.

vided $K<1 / \mu s$. It is interesting that for fixed $\mu$ the expected extinction time declines as the selection coefficient increases. This is in contrast to the usual view that Muller's ratchet does more damage when $s$ is small (Maynard Smith, 1978; Bell, 1988; Kondrashov, 1988). The ratchet does click less frequently as $s$ increases, but this is more than compensated for by the greater reduction in survivorship per click.

Third, for $R=2$ and a low mutation load $(\mu s)$ and/or high mutation pressure $(\mu)$, there is a broad range of $K$ over which the expected extinction time increases slowly. This is qualitatively consistent with the observation that asexual protozoan and tissue cultures have characteristic extinction times. The results are also quantitatively consistent with the fact that the longevity of such cultures rarely ever exceeds a few thousand cell divisions.

Back-mutations may rescue extremely large populations, such as bacteria, from the melt-down process that we are modeling. Roughly speaking, this would require that back-mutations have a high likelihood of occurring prior to the expected extinction time in their absence, i.e., $K \bar{t}_{\mathrm{E}} \gg 1 / \mu^{*}$ where $\mu^{*}$ is the back-mutation rate. With a low mutation load $(\mu s=0.0002)$ and $R=2, \bar{t}_{\mathrm{E}}$ asymptotes at approximately $4 \times 10^{3}$, so for a population size of $10^{8}$ the critical back- mutation rate would be $2.5 \times 10^{-12}$, which is quite conceivable.

If the mutation pressure is high and the intensity of selection is low relative to the magnitude of random genetic drift, the distribution of extinction times can be mod-

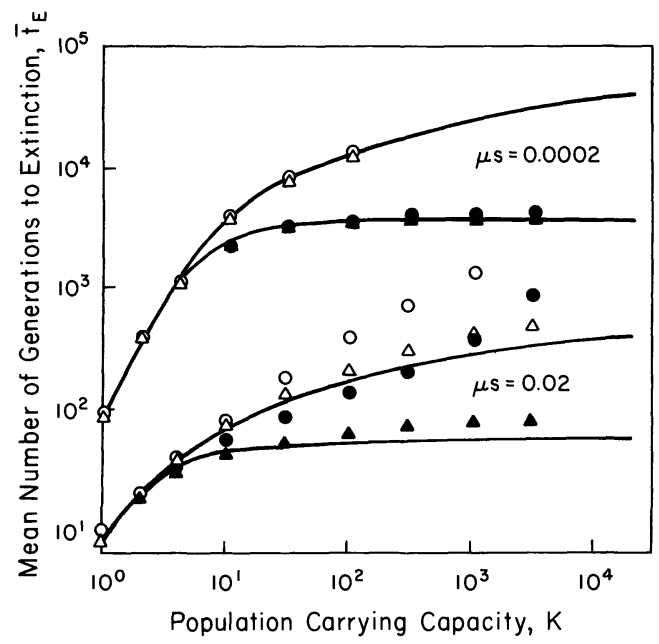

FIG. 6. Expected extinction time as a function of population carrying capacity under asexuality. Upper and lower sets of points refer to mutational loads of $\mu \mathrm{s}$ $=0.0002$ and 0.02 . Open points denote $R=K$ (high fecundity) and closed points denote $R=2$. Circles and triangles denote low $(\mu=0.3)$ and high $(\mu=1.2) \mathrm{mu}-$ tation pressure. The curves are the deterministic solutions described in the text. 


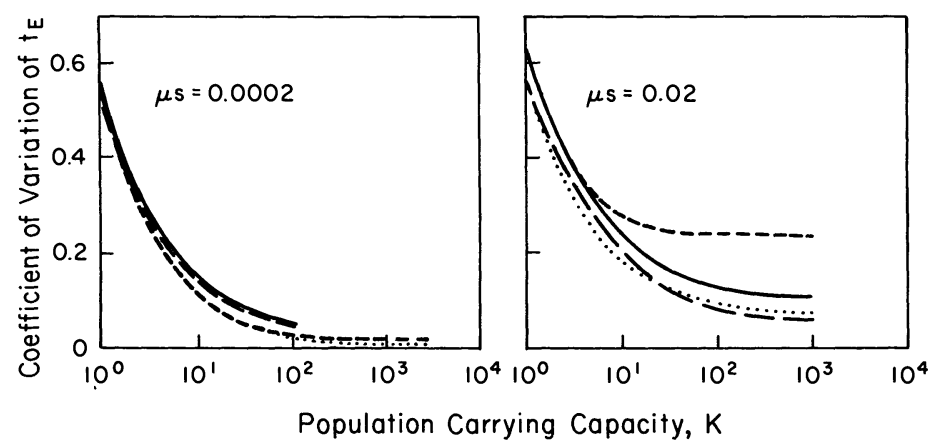

Fig. 7. Coefficients of variation of extinction times obtained by simulation. Legend: solid line, $R=K, \mu=$ 0.3 ; large dashes, $R=K, \mu=1.2$; small dashes, $R=2, \mu=0.3$; dotted line, $R=2, \mu=1.2$.

eled in an essentially deterministic fashion. Under these conditions, the expected number of mutations per individual at time $t$ is approximately $\mu t$, so the mean probability of individual survival is $\overline{W_{t}} \simeq(1-s)^{\mu t}$. The expected population size at the beginning of generation $t$ is $N_{t} \simeq R N_{t-1} \overline{W_{t-1}} \leq K$, and the probability of a population extinction occurring in generation $t$ is $1-P_{t} \simeq(1-$ $\left(\bar{W}_{t}\right)^{N_{t}}$. The fraction of replicate populations surviving to generation $x$ is $l_{x} \simeq \prod_{t=0}^{x-1} P_{t}$. Iteration of these expressions until $l_{x}=0.5$ gives the median longevity of populations. Figure 6 illustrates that these projections are very close to the mean extinction times obtained by direct simulation provided $K<$ $\mu / s$.
The coefficient of variation of extinction times is generally quite low except at very small $(K<10)$ population sizes (Fig. 7). When the mutation load is weak, $C V\left(t_{\mathrm{E}}\right)<$ 0.1 for the entire range of $\mu$ and $R$ provided $K>30 . C V\left(t_{\mathrm{E}}\right)$ is higher when the load is high, but still less than 0.2 provided $K>$ 100 so long as the genomic mutation rate is high. These results are again broadly compatible with the characteristic life-spans often associated with laboratory cultures of protozoans and fibroblasts.

For the most part, our results bear out the common notion that Muller's ratchet is ineffective in sexual populations. For "single-seed-descent" situations $(R=K=1)$, self-fertilization extends the longevity of

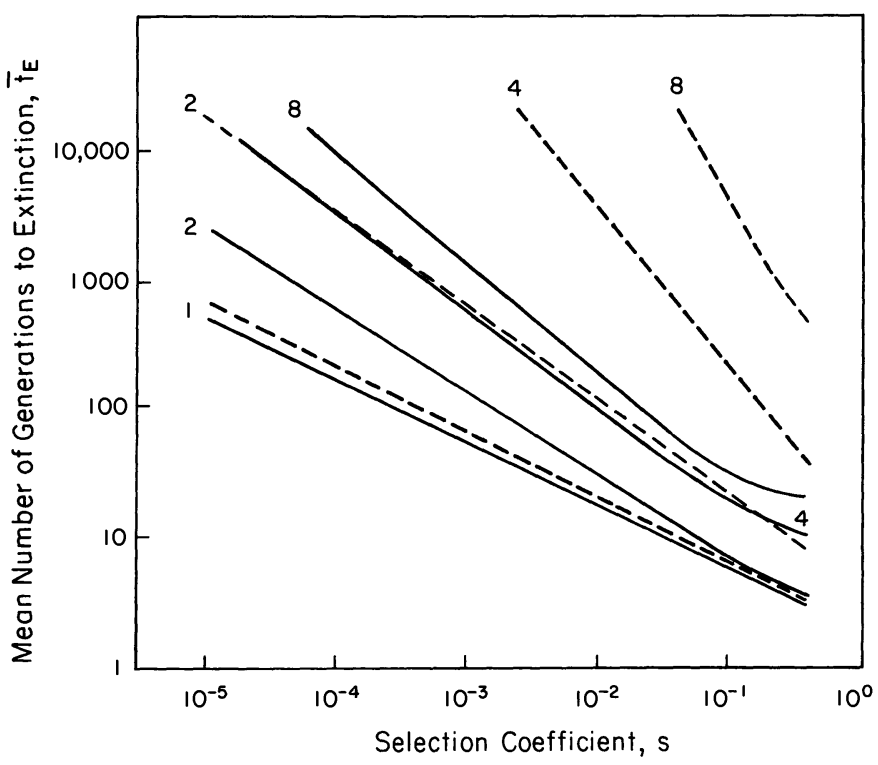

FIG. 8. Expected times to extinction as a function of the selection coefficient for population sizes of 1, 2, 4, and $8(R=K)$ under sexuality (dashed lines) and asexuality (solid lines). The mutation rate is $0.6 /$ zygote. 

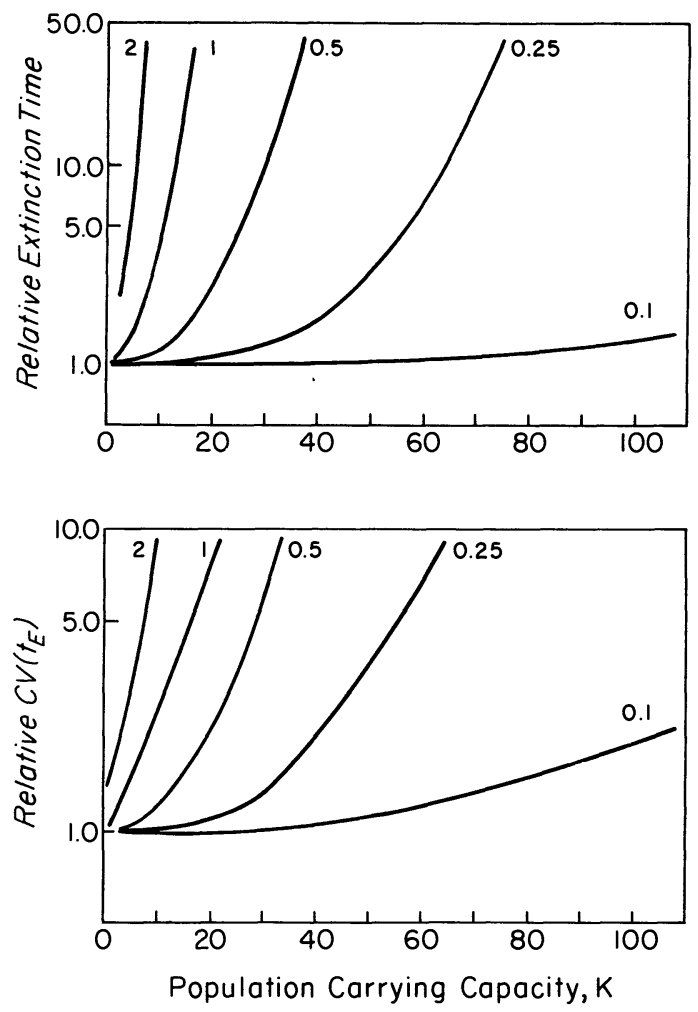

FIG. 9. Ratio of expected extinction times when mutations have variable effects relative to when mutational effects are constant, and the analogous ratios for coefficients of variation of extinction times. The numbers on the curves are the coefficients of variation of selection coefficients. In all cases, $\mu=0.3, \bar{s}=0.0067$, and $R=K$.

lineages by $10-20 \%$ relative to asexuality (Fig. 8). A full-sib mated lineage has an expected survival time three to six times that of a clonal lineage with $K=2$. With high fecundity, sex extends the mean extinction time by an order of magnitude at $K=4$ and by two orders of magnitude at $K=8$. Simulations beyond $K=8$ are enormously timeconsuming due to the exponential increase in $t_{\mathrm{E}}$ with $K$ in sexual populations.

\section{COMPENSATORY MUTATION}

In the preceding analyses, it has been assumed that all mutations are unconditionally deleterious with constant effect $s$. In reality, however, there will be some variance for mutational effects, and some mutations may be beneficial. If we assume that the average reduction in fitness per mutation $(\bar{s})$ is independent of the genetic background, variance in $s$ suggests the possibility that the reduction in fitness caused by a mutation at one locus may be compensated by a beneficial mutation at another locus. The effective mutation load is reduced with variable mutation effects because mutations that are much more deleterious than average are rapidly eliminated while those that are mildly favorable can go to fixation.

In the appendix we outline a simple model that allows for compensatory mutation while ensuring that the effects of mutations are deleterious on average. The main features of this model are (1) the mean and variance of mutational effects on fitness are independent of the genetic background, and (2) the mutational process cannot produce a genotype that is better than the initial optimum [if $W$ is an individual's current fitness, this constrains the range of mutational effects to be $1-(1 / W) \leq s \leq 1]$ so that offspring fitness is always in the range $(0,1)$.

Variance in mutational effects enhances the longevity of asexual lineages (Fig. 9). The magnitude of this enhancement increases at a more than exponential rate with $K$ and is quite pronounced even at relatively small population sizes provided the coefficient of variation of $s$ is greater than 0.5 . On the other hand, if the coefficient of variation of $s$ is less than 0.1 , the effect is negligible until moderately large $K$ are reached. Not surprisingly, variance in mutational effects also inflates the variance in extinction times (Fig. 9).

\section{DisCUSSION}

Although there have been many previous attempts to evaluate the joint effects of selection and mutation in finite populations, all of these studies have effectively decoupled the dynamics of gene frequency change and population size by treating the latter as a constant. With this approach, it is possible to obtain expressions for the equilibrium genetic load, the steady-state rate of accumulation of mutations, etc. However, we have shown that when small populations (especially asexual ones) incur deleterious viability mutations, the mutation load does not attain an equilibrium. As mutations accumulate, the number of reproducing adults declines. This causes a temporal increase in 
the relative importance of random genetic drift, which increases the likelihood of fixing future deleterious mutations. The eventual result of this synergistic interaction is population extinction. Although we have focused on viability mutations, we see no reason why the results should not extend to mutations affecting progeny production.

Escape from the mutational melt-down requires an appreciable incidence of backmutation or a reliable repair mechanism. In sexual organisms, segregation and recombination provide an efficient means of eliminating deleterious mutations since parents can always produce progeny with elevated fitness. Nevertheless, mutational meltdowns can occur in very tiny sexual populations. They are inevitable in obligately selffertilizing lineages, which indicates that obligate self-fertilization is not a stable evolutionary strategy. Such a view is consistent with the existing data on plant mating systems (Schemske and Lande, 1985). Although some deleterious mutations can become fixed by chance in larger sexual populations, the prolongation of population life-span accomplished by segregation and recombination will greatly increase the opportunity for back-mutation, thereby providing a secondary means of maintaining the mutation load at a low level.

For obligately asexual species, avoidance of a mutational melt-down requires some form of back-mutation. The conventional view is that the rate of back-mutation to a normal allele is at least two orders of magnitude less than the forward rate of mutation to deleterious alleles (Maynard Smith, 1978). Under these conditions, locus-specific back-mutation can do little to stop the operation of Muller's ratchet, except in enormous populations. However, compensatory mutations of other loci influencing the same or different trait may lead to a substantial amount of effective back-mutation for fitness (Gabriel and Wagner, 1988). This is because selection sorts individuals on the basis of the composite properties of the entire phenotype regardless of the genotypic states at individual loci (Lynch and Gabriel, 1983). Consider, for example, a trait such as body size under stabilizing selection. An upward mutation that causes a deleterious increase in size can be bal- anced by a downward mutation at any locus affecting size or by a mutation that influences another character in some way that compensates for abnormally large size.

Taking this approach, we have shown that the mean extinction time can be increased dramatically when the effects of mutations are highly variable relative to their expectations. Thus variance in mutation effects acts as a sort of repair mechanism. It would be very interesting to know whether such a simple mechanism might be responsible for the abnormally high longevities of cultures of human HeLa cells and amicronucleate Tetrahymena, both of which are often described as immortal.

An inherent problem with mutations of small effects is that they are exceedingly difficult to study on an individual basis. Except for the results from several large mutation accumulation experiments involving Drosophila melanogaster chromosomes (Mukai, 1979; Crow and Simmons, 1983), there are almost no data on the mutation load in the literature. To these we have added estimates derived from some unconventional systems (ciliated protozoans and in vitro cultures of vertebrate fibroblasts). While the mutational mechanisms responsible for the degradation in fitness may differ in these three systems, it is worth noting that the average estimated viability mutational loads are of the same order of magnitude $(0.015$ for Drosophila, 0.01 for mammalian and avian fibroblasts, and 0.005 for ciliates).

In deriving these estimates, we illustrated several potential empirical approaches for measuring the effective mutation load. For asexual species, the simplest approach appears to be through the use of "single-seeddescent" experiments. A large number of lines (preferably in excess of 100) derived from a single mother are maintained in isolation by passing single random progeny from each line on to the next generation. Each death prior to maturity marks the extinction of a line. With $K=R=1$, there is no opportunity for selection within lines, so viability mutations accumulate in a neutral fashion. Letting $W(t)$ be the fraction of extant lines at time $t$ that survive to $t+1$, then $\ln [W(t)]=\ln \left(W_{0}\right)+[\mu \ln (1-s)] t \simeq$ $\ln \left(W_{0}\right)-\mu s t$. Thus, a least-squares regression of $\ln [W(t)]$ on $t$ yields an estimate of 
the effective mutation load. Ideally, the points in this regression should be weighted by the inverse of the sampling variance of $\ln [W(t)]$, i.e., by $L(t) W(t) /[1-W(t)]$ where $L(t)$ is the number of surviving lines at time $t$. Care must be taken in attaching a standard error to the regression coefficient; since the data points are not independent, the usual expression does not apply (Lynch, 1988). Such experiments can also profit from the evaluation of parallel controls, such as progeny drawn from a pool of stored seed or from a large panmictic population. By treating the control observations as covariates, it is possible to eliminate much of the noise in the estimates of $W(t)$ resulting from temporal changes in the environmental sources of mortality (Lynch, 1988).

These same procedures can be followed with self-compatible sexual species by enforcing self-fertilization within each line. The main limitation in this case is the need to start with pure lines so as not to inflate the initial mortality of lines by the segregation of deleterious recessive alleles that are heterozygous in the base population. Such lines may be obtained by imposing several preliminary generations of self-fertilization. In many plant species, it is now possible to rapidly produce completely homozygous lines (doubled haploids) by cytological manipulation.

\section{ACKNOWLEDGMENTS}

This work has been supported by NSF Grant BSR 86-00487 and PHS Grant R01 GM36827-01 to M.L. and by a grant from Deutsche Forschungsgemeinschaft to W.G. Many thanks to R. Bürger, L. Chao, J. Drake, and D. Nanney for helpful comments.

\section{Literature Cited}

Angello, J. C., AND J. W. Prothero. 1985. Clonal attenuation in chick embryo fibroblasts. Cell Tissue Kinet. 18:27-43.

AufDERHEIDE, K. J. 1984. Clonal aging in Paramecium tetraurelia. I. Absence of evidence for a cytoplasmic factor. Mech. Ageing Dev. 28:57-66.

- 1987. Clonal aging in Paramecium tetraurelia. II. Evidence of functional changes in the macronucleus with age. Mech. Ageing Dev. 37:265279.

BeLL, G. 1982. The Masterpiece of Nature: The Evolution and Genetics of Sexuality. Univ. Calif. Press, Berkeley.
1988. Recombination and the immortality of the germ line. J. Evol. Biol. 1:67-82.

CHAO, L. 1988. Evolution of sex in RNA viruses. J. Theoret. Biol. 133:99-112.

Crow, J. F., AND M. KIMURA. 1970. An Introduction to Population Genetics Theory. Harper \& Row, N.Y.

Crow, J. F., AND M. J. Simmons. 1983. The mutation load in Drosophila, pp. 2-35. In M. Ashburner, H. L. Carson, and J. N. Thompson, Jr. (eds.), The Genetics and Biology of Drosophila, Vol. 3C. Academic Press, N.Y.

FelsensteIn, J. 1974. The evolutionary advantage of recombination. Genetics 78:737-756.

Gabriel, W., AND G. P. Wagner. 1988. Parthenogenetic populations can remain stable in spite of high mutation rate and random drift. Naturwissenschaften 75:204-205.

HaldaNe, J. B. S. 1937. The effect of variation on fitness. Am. Natur. 71:337-349.

HAYFLICK, L. 1984. Intracellular determinants of cell aging. Mech. Ageing Dev. 28:177-185.

Heller, R., AND J. MaYnard Smith. 1978. Does Muller's ratchet work with selfing? Genet. Res. 32: 289-293.

Hopf, F. A., R. E. Michod, AND M. J. SANDERSON. 1988. The effect of the reproductive system on mutation load. Theoret. Popul. Biol. 33:243-265.

Karatza, C., AND S. Shall. 1984. The reproductive potential of normal mouse embryo fibroblasts during culture in vitro. J. Cell Sci. 66:401-409.

KIMURA, M. 1957. Some problems of stochastic processes in genetics. Ann. Math. Stat. 28:882-901.

Kimura, M., T. Maruyama, and J. F. Crow. 1963. The mutation load in small populations. Genetics 48:1303-1312.

KoNDRASHOV, A.S. 1988. Deleterious mutations and the evolution of sexual reproduction. Nature (London) 336:435-440.

LANDE, R. 1988. Genetics and demography in biological conservation. Science 241:1455-1459.

LYNCH, M. 1984. Destabilizing hybridization, general-purpose genotypes and geographic parthenogenesis. Quart. Rev. Biol. 59:257-290.

1988. Design and analysis of experiments on random drift and inbreeding depression. Genetics 120:791-807.

LYNCH, M., AND W. GABRIEL. 1983. Phenotypic evolution and parthenogenesis. Am. Natur. 122:745764.

Maynard Smith, J. 1978. The Evolution of Sex. Cambridge Univ. Press, Cambridge, UK.

Michod, R. E., AND B. R. LEVIN (eds.). 1988. The Evolution of Sex. Sinauer Associates, Sunderland, MA.

MuKAI, T. 1979. Polygenic mutations, pp. 177-196. In J. N. Thompson, Jr. and J. M. Thoday (eds.), Quantitative Genetic Variation. Academic Press, N.Y.

MulleR, H. J. 1950. Our load of mutations. Am. J. Hum. Genet. 2:111-176.

1964. The relation of recombination to mutational advance. Mutat. Res. 1:2-9.

NANNEY, D. L. 1974. Aging and long-term temporal regulation in ciliated protozoa: A critical review. Mech. Ageing Dev. 3:81-105. 
NEI, M. 1968. The frequency distribution of lethal chromosomes in finite populations. Proc. Natl. Acad. Sci. U.S.A. 60:517-524.

- 1983. Genetic polymorphism and the role of mutation in evolution, pp. 165-190. In M. Nei and R. K. Koehn (eds.), Evolution of Genes and Proteins. Sinauer Associates, Sunderland, MA.

Pamilo, P., M. NeI, AND W.-H. LI. 1987. Accumulation of mutations in sexual and asexual populations. Genet. Res. 49:135-146.

Raes, M., AND J. REMACle. 1983. Ageing of hamster embryo fibroblasts as the result of both differentiation and stochastic mechanisms. Exp. Gerontol. 18:223-240.

SCHEMSKe, D. W., AND R. LANDE. 1985. The evolution of self-fertilization and inbreeding depression in plants. II. Empirical observations. Evolution 39:41-52.

Simon, E. M., AND D. L. NANNEY. 1979. Germinal aging in Tetrahymena thermophila. Mech. Ageing Dev. 11:253-268.

Smith, J. R., AND O. Pereira-Smith. 1977. Colony size distribution as a measure of age in cultured human cells. A brief note. Mech. Ageing Dev. 6: 283-286.

SMITH-SONNEBORN, J. 1979. DNA repair and longevity assurance in Paramecium tetraurelia. Science 203:1115-1117.

- 1981. Genetics and aging in protozoa. Int. Rev. Cytol. 73:319-354.

SONNEBORN, T. M. 1954. The relationship of autogamy to senescence and rejuvenescence in Paramecium aurelia. J. Protozool. 1:38-53.

- 1957. Breeding systems, reproductive methods and species problems in protozoa, pp. 155-324. In E. Mayr (ed.), The Species Problem. Amer. Assoc. Adv. Sci., Washington, D.C.

Soulé, M. E. (ed.). 1987. Viable Populations for Conservation. Cambridge Univ. Press, Cambridge, UK.

Stanulis-Praeger, B. M. 1987. Cellular senescence revisited: A review. Mech. Ageing Dev. 38:1-48.

TAKAGI, Y., AND M. YoshidA. 1980. Clonal death associated with the number of fissions in Paramecium caudatum. J. Cell Sci. 41:177-191.

voN MAGNUS, P. 1954. Incomplete forms of influenza virus. Adv. Virus Res. 2:59-79.

WAllace, B. 1987. Fifty years of genetic load. J. Hered. 78:134-142.

Corresponding Editor: A. G. Clark

\section{APPENDIX}

To allow for the compensatory effects of mutations, we let the expected proportional reduction in fitness with each new mutation be $\bar{s}$. However, the realized fitness of each new mutant varies around its expectation with fixed variance $\sigma_{W}^{2}$. A mutation is compensatory whenever $W^{\prime}>W$, where $W$ and $W^{\prime}$ are an individual's fitness before and after mutation. As a boundary condition, we impose $W^{\prime} \leq 1$, so that mutation never creates a genotype with fitness in excess of the original optimum.

This type of distribution of mutational effects can be implemented in the following way. We assume that fitness is determined by an underlying variable $z$, such that

$$
W(z)=\exp (-z)
$$

Since

$$
W\left(z^{\prime}\right)=(1-s) W(z)
$$

it follows that

$$
\Delta z=z^{\prime}-z=-\ln (1-s)
$$

Thus, for a given selection coefficient $(s)$, the mutational effect on the underlying scale is independent of the prior phenotypic value. The assumed multiplicative effects of deleterious mutations

$$
W=\prod_{\imath=1}^{n}\left(1-s_{\imath}\right)
$$

transform into additive effects on the underlying scale

$$
W(z)=\exp \left(-\sum_{\imath=1}^{n} \Delta z_{\imath}\right)
$$

To ensure that the mutational process does not produce supervital individuals $\left(W^{\prime}>1\right)$, we require that $\sum_{t=1}^{n} \Delta z_{l} \geq 0$. This is accomplished by letting $z^{\prime}$ have a conditional gamma distribution with parameters $a$ and $p$. We then have

$$
\begin{aligned}
E\left[W\left(z^{\prime} \mid z\right)\right] & =\int_{0}^{\infty} \exp (-x)\left[\frac{a^{p}}{\Gamma(p)} x^{p-1} \exp (-a x)\right] d x \\
& =\left(\frac{a}{a+1}\right)^{p} \\
E\left[W^{2}\left(z^{\prime} \mid z\right)\right] & =\int_{0}^{\infty} \exp (-2 x)\left[\frac{a^{p}}{\Gamma(p)} x^{p-1} \exp (-a x)\right] d x \\
& =\left(\frac{a}{a+2}\right)^{p} \\
\sigma_{W^{2}} & =E\left[W^{2}\left(z^{\prime} \mid z\right)\right]-E^{2}\left[W\left(z^{\prime} \mid z\right)\right]
\end{aligned}
$$

With our requirements that $E\left[W\left(z^{\prime} \mid z\right)\right]=(1-\bar{s}) W(z)$ and that $\sigma_{W}^{2}$ is fixed and independent of $z$, we have two equations for the unknown parameters $a$ and $p$. Since no analytical solution exists for the exponential equations, the parameters have to be calculated numerically. A solution exists only if $E\left[W^{2}\left(z^{\prime} \mid z\right)\right]<1$. This places an upper limit on the coefficient of variation of mutational effects that can be studied with this model,

$$
\frac{\sigma_{W}}{\bar{s}}<\left(\frac{2}{\bar{s}}-1\right)^{1 / 2}
$$

